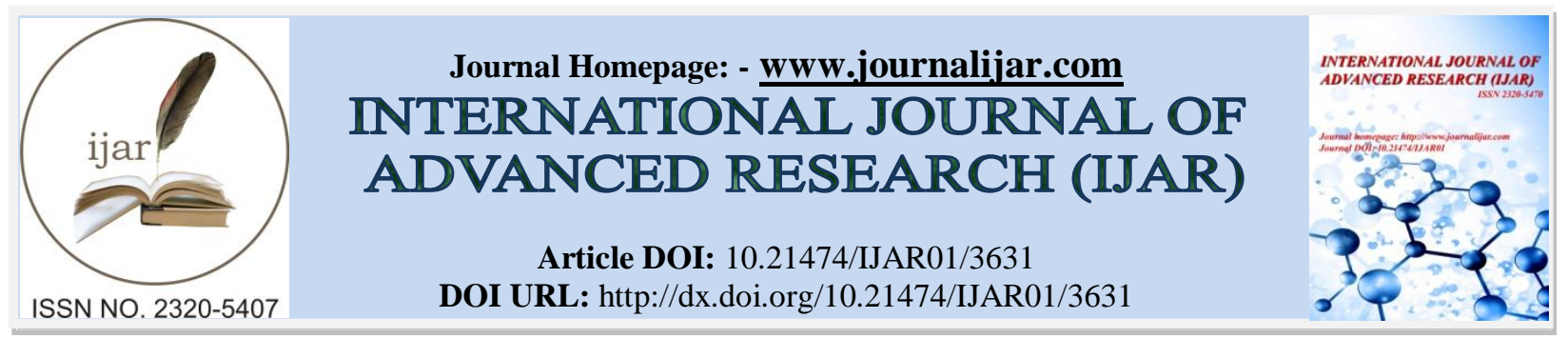

RESEARCH ARTICLE

\title{
ESTIMATION OF HEART RATE USING SIGNAL FUSION OF ECG AND BP SIGNALS
}

Bhargavi B. G. S, Devi Nallammai RM and Palani Thanaraj. K.

Department of Electronics and Instrumentation Engineering, St.Joseph's College of Engineering, Chennai-600119, Tamilnadu, India.

\section{Manuscript Info}

Manuscript History

Received: 08 January 2017

Final Accepted: 03 February 2017

Published: March 2017

Key words:-

signal fusion, ECG, BP, heart rate, MSE, SNR.

\begin{abstract}
The main aim of this paper is to estimate the heart rate and ECG pattern of a person as obtained from a polysomnographic record for detection of respiration related sleep disorders by fusing the data contained from the ECG and BP waveforms.. Polysomnography records the brain waves, the oxygen level in the blood, heart rate and breathing, as well as eye and leg movements during the study Data fusion can be defined as a combination of data from multiple sources to obtain improved information, meaning less expensive, higher quality, or more relevant information. For this study two specific signals from the polysomnogram is chosen- ECG and Blood pressure (BP). They are fused to obtain the improved heart rate of the subjects. Initially the individual heart rates are computed and compared against the heart rate of the fused signal. In case of noisy measurement environments, this method can be implemented.
\end{abstract}

Copy Right, IJAR, 2017,. All rights reserved.

Introduction:-

Polysomnography is a sleep study. This test records certain body functions as we sleep, or try to sleep. Polysomnography is used to diagnose sleep disorders. A change in the cycle may make it hard for a person to sleep soundly. Multiple sleep latency test (MSLT) test measures how long it takes for us to fall asleep. It also determines whether you enter REM sleep [1]. Maintenance of wakefulness test (MWT) measures whether we can stay awake during a time when we are normally awake. If shift work sleep disorder or another problem with our body's internal clock (circadian rhythm) is suspected, then the test performed is called actigraphy.

Among the three main tests suggested above, polysomnography is perhaps the most elaborate and time consuming, due to the sheer amount of data collected and stored .But this is also the most valuable and reliable test in terms of the accuracy of sleep information obtained, provided they can be analyzed and interpreted in the clinically correct manner. Signal fusion involves the consolidation of all the various signals obtained in the polysomnogram in such a constructive manner so that it can be used constructively for diagnosis of sleep disorders.

Signal processing is an enabling technology that encompasses the fundamental theory, applications, algorithms, and implementations of processing or transferring information contained in many different physical, symbolic, or abstract formats broadly designated as signals. It uses mathematical, statistical, computational representations and techniques for representation, modeling, analysis, synthesis, discovery, recovery, sensing, acquisition, extraction, learning, security, or forensics. Dynamical mathematical model in this context is a mathematical description of the dynamic behavior of a system or process in either the time or frequency domain 
Biomedical signals are observations of physiological activities of organisms, ranging from genetic factor and protein sequences, to neural and cardiac rhythms, to tissue and organ images. Biomedical signal processing aims at extracting significant information from biomedical signals. With the help of biomedical signal processing, biologists can discover new methods of detection and physicians can monitor distinct illnesses. Initially the primary focus of biomedical signal processing was on filtering signals to remove noise. Sources of noise arise from imprecision of instruments to interference of power lines. Other sources are due to the biological systems themselves under study. The analysis framework derives its basics from statistical signal processing. This framework treats the data as random signals; the processing, e.g. Wiener filtering or Kalman filtering which utilize statistical characterizations of the signals to extract desired signal components.

Our bodies are constantly communicating information about our health. This information can be captured through physiological instruments that measure heart rate, blood pressure, oxygen saturation levels, blood glucose, nerve conduction, brain activity and so forth. Traditionally, such measurements are taken at specific points in time and noted on a patient's chart. Physicians actually see less than one percent of these values as they make their rounds - and treatment decisions are made based upon these readings.

Biomedical signal processing involves the analysis of these measurements to provide useful information upon which clinicians can make decisions. Engineers are discovering new ways to process these signals using a variety of mathematical formulae and algorithms. Working with traditional bio-measurement tools, the signals can be computed by software to provide physicians with real-time data and greater insights to aid in clinical assessments. By using more refined means to analyze what our bodies are saying, we can potentially determine the state of a patient's health through more noninvasive measures. Our proposed system works in this direction with further emphasis on processing the obtained data to a certain level so that it gives robust estimates of the patient's medical condition.

Sleep is defined as a reversible behavioral state, which involves a substantial form of disengagement and a decrease in the responsiveness to the environment. There are two types of sleep: Non rapid eye movement sleep (NREM) and rapid eye movement sleep (REM). NREM sleep is divided into four types based on the EEG signals. REM sleep is characterized by an increased activity in the EEG which could be a result of muscle atonia in the skeletal muscles or due to short episodic bursts of the REM sleep.

Polysomnography is used to diagnose sleep disorders. A change in the cycle may make it hard for a person to sleep soundly. Channels commonly recorded during a PSG are: Brain wave activity (EEG), Eye movement (EOG), Muscle tone (chin EMG),Airflow via thin catheters placed in front of nostrils and mouth, heart rhythm (EKG),Oxygen level (SpO2),Leg muscle activity (PLM),Body position, Video recording. All the obtained information in this test can be used suitably for various purposes. This paper is one such application.

\section{Materials and Methods:-}

Estimation of respiratory rate [8] from waveforms recorded from passively breathing subjects is notoriously difficult, due in part to the non-stationary nature of the signals and in part the frequent non-stationary noise . Methods for recoding a time series of respiratory effort include impedance pneumography, impedance plethysmograpy and flow thermography which measures the changes in temperature of air flow as it moves in and out of the mouth and/or nose over a thermistor. Literature on deriving a respiratory signal from other related signals is dense in the case of the electrocardiogram, but relatively less for other signals such as the photo plethysmogram and blood pressure waveform [2].The field of respiration rate estimation from respiratory signals (whether derived or not) is scantily covered in the public literature, particularly with respect to large data sets. Key works by O'Brien and Heneghan, de Chazal et al., Ishijima, Park et al., and Tarassenko [15] et al. highlights the importance of modeling noise and combining information from multiple ECG leads and sensor modalities to compensate for noisy measurements. To our knowledge, very little work has been public.

To evaluate our methods we have used polysomnographic data from a polysomnographic database obtained from www. physionet.org/cgi-bin/atm/ATM. it consists of the four most basic records namely- ECG, BP, EEG and respiratory rate. In this study we are concerned with the ECG [3] and BP [4] signals only. The records of five patients have been studied. The relation between the above mentioned signals is arrived at by the results of the correlation table presented below [5]. Out of the three signals, the most meaningfully correlated are the ECG and BP [6]. 
From these two signals the respective heart rates are obtained through a heart rate algorithm. This mainly counts the number of R peals in an ECG. In the case of a BP signal [7], each peak corresponding to every cardiac cycle is counted. This provides the heart rate of the person. This procedure can also be followed for signals with noise. For a more realistic approximation we have added noise of different SNR values $(10 \mathrm{~dB}, 20 \mathrm{~dB}, 30 \mathrm{~dB}, 40 \mathrm{~dB}$ and $50 \mathrm{~dB})$.

Table 1: Correlation between signals.

\begin{tabular}{|c|c|c|c|}
\hline ECG & BP & EEG & RR \\
\hline slp01 & 0.0736 & 0.1391 & 0.004 \\
\hline slp14 & 0.0983 & 0.1163 & 0.0098 \\
\hline slp37 & -0.0962 & 0.3556 & 0.0237 \\
\hline slp41 & 0.1269 & 0.2456 & -0.0134 \\
\hline slp59 & 0.0545 & -0.0441 & -0.0055 \\
\hline slp67x & 0.0427 & -0.2804 & -0.0083 \\
\hline
\end{tabular}

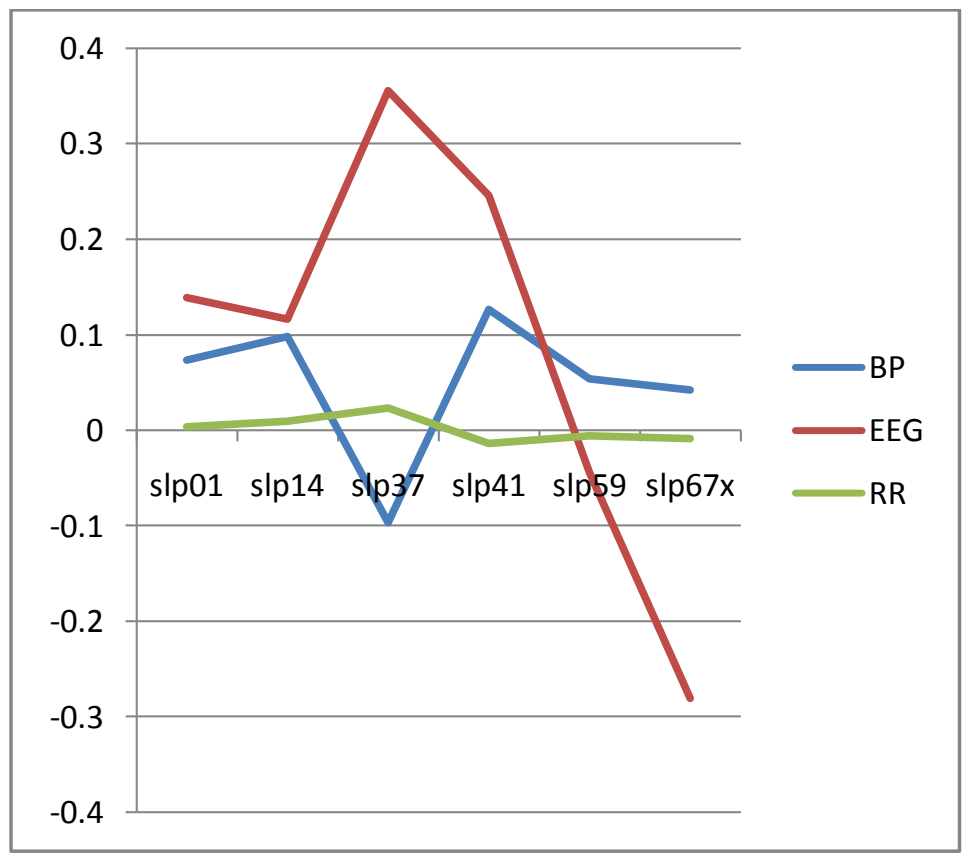

Fig 1: A plot of the correlation table

The main steps involved are presented in the block diagram given below. Both the required signals are obtained from the database. The signals in the database are clean and not corrupted by external noise. The heart rate for this is determined and this acts as the standard for comparison after fusion of the signals. The working data set is obtained by adding suitable noise to the signals. This noisy data is fused by the signal fusion algorithm and the output displayed is of a noiseless fused signal. The heart rate of this signal is computed by the beat detector algorithm and compared against the heart rate value of the reference signal [14].

The beat detection algorithm used is the Hooman Sedghamiz peak detector [9]. This algorithm requires the ECG as a signal vector along with the sampling frequency and the time period for the output window. As the output, it computes the indexes and amplitudes of each wave in the component ECG [8], the estimated heart rate and displays the processed signals. The function used is as given below

[R_i,R_amp,S_i,S_amp,T_i,T_amp,Q_i,Q_amp,heart_rate,buffer_plot]=peakdetect(ECG,fs,view) 
Data fusion [10] is a multidisciplinary area that involves several fields, and it is difficult to establish a clear and strict classification. The fusion of these two signals is brought about by a fusion algorithm [11]. It is based on a kalman filter fusion that merges both the signals as one .this is further used for heart rate comparison after fusion.

The Kalman filter is the most popular estimation technique [9]. It was originally proposed by Kalman and has been widely studied and applied since then.

The Kalman filter estimates the state $x$ of a discrete time process governed by the following space-time model:

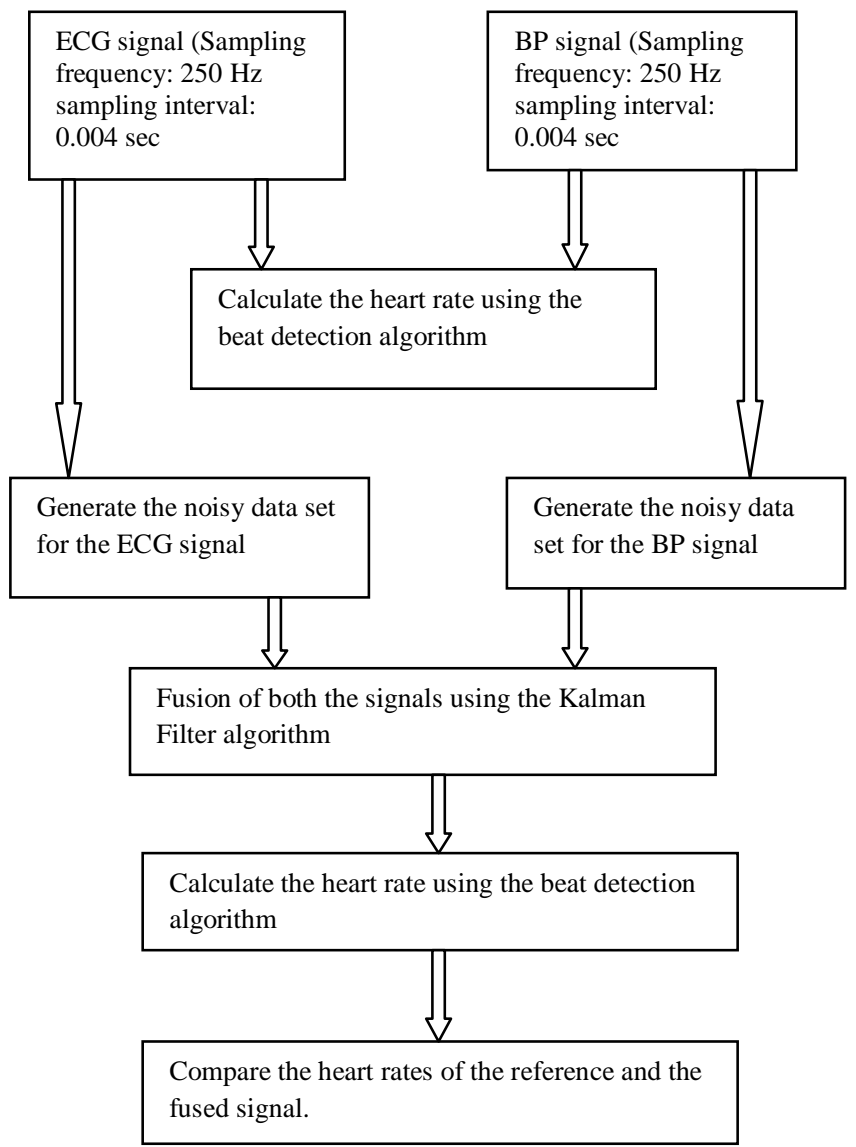

Fig 2: block diagram of the process

$$
x(k+1)=\Phi(k) x(k)+G(k) u(k)+w(k)
$$

With the observations or measurements zat time $k$ of the state $x$ represented by

$$
Z(k)=H(k) x(k)+V(k)
$$

Where $\Phi(k)$ is the state transition matrix, $G(k)$ is the input matrix transition, $u(k)$ is the input vector, $H(k)$ is the measurement matrix, and wand $\mathrm{V}$ are the random Gaussian variables with zero mean and covariance matrices of $Q(k)$ and $R(k)$, respectively.

The Kalman filter [12] is mainly employed to fuse low-level data. If the system could be described as a linear model and the error could be modeled as the Gaussian noise, then the recursive Kalman filter obtains optimal statistical estimations. However, other methods are required to address nonlinear dynamic models and non linear measurements . The filter as used in this method is given as: 
For single signal:

xhat = kalman $(\mathrm{z} 1, \mathrm{~A}, \mathrm{C} 1, \mathrm{R} 1, \mathrm{Q})$;

For two signals:

xhat = kalman([z1; z2], A, [C1; C2], [R1 0; 0 R2], Q);

Where:

xhat $=$ estimated signal

$\mathrm{Z} \quad$ = input signal

$\mathrm{A}=$ state transition model

$\mathrm{C}=$ observation model

$\mathrm{R}=$ measurement noise covariance

$\mathrm{Q}=$ process noise covariance

Fig 3: A pure ECG signal

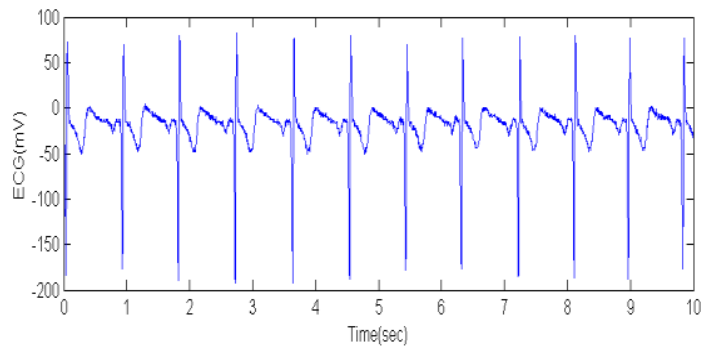

Fig 4: The same signal with a noise of $20 \mathrm{db}$ added

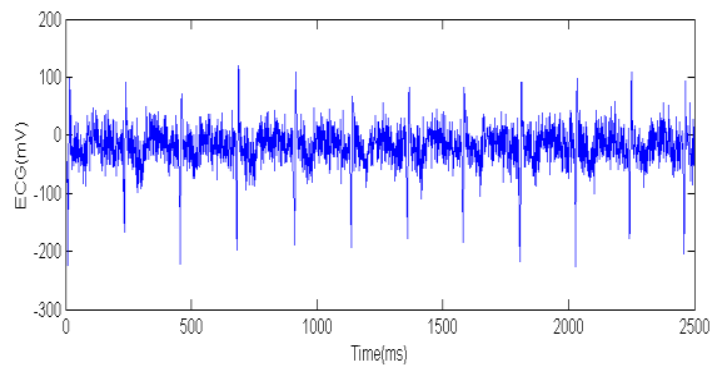

Fig 5: With 40db noise

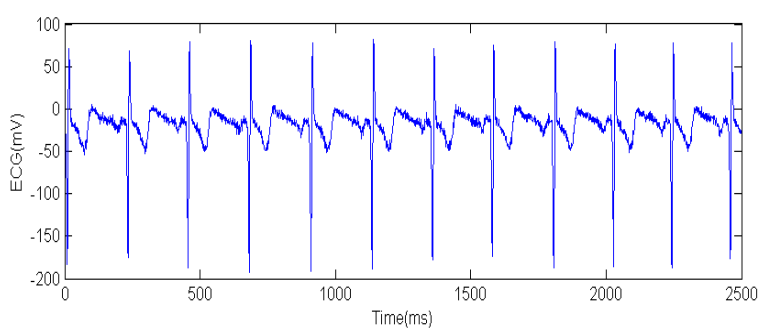


Fig 6: The heart rate obtained from the ECG signal
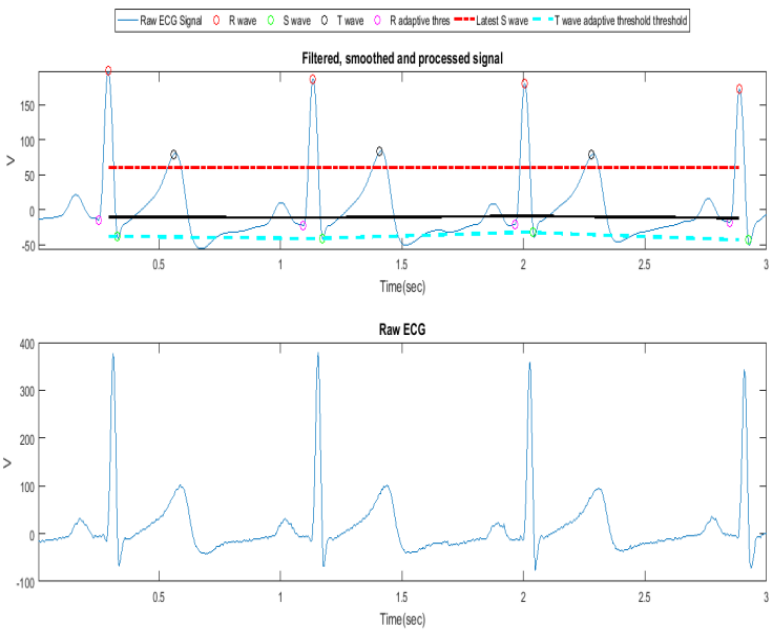

Fig 7: A pure BP signal

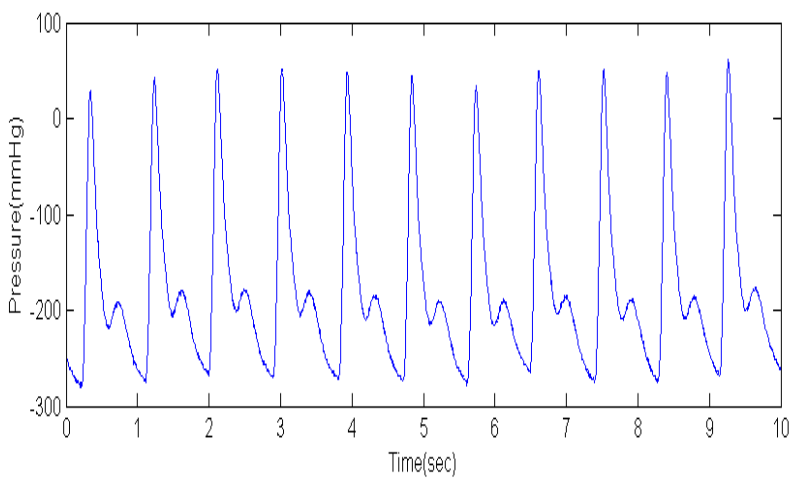

Fig 8: The same signal with 20db noise

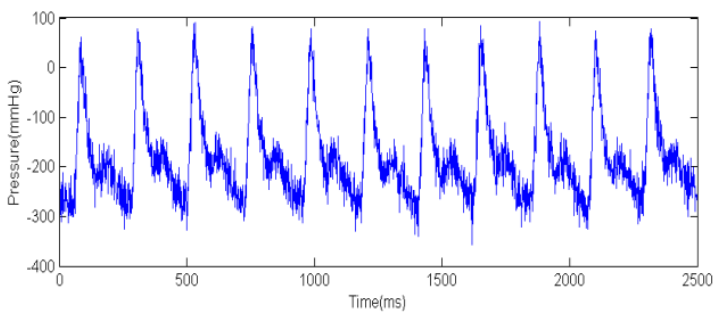

Fig 9: With 40db noise

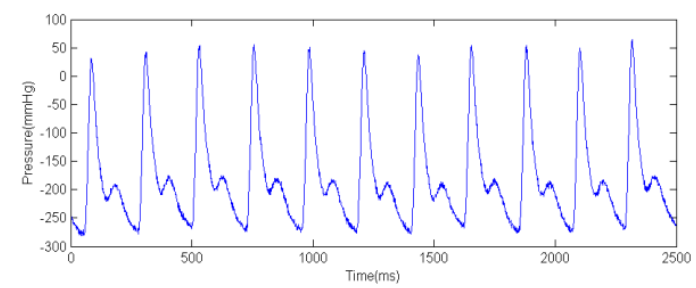


Fig 10: The heart rate obtained from the BP signal
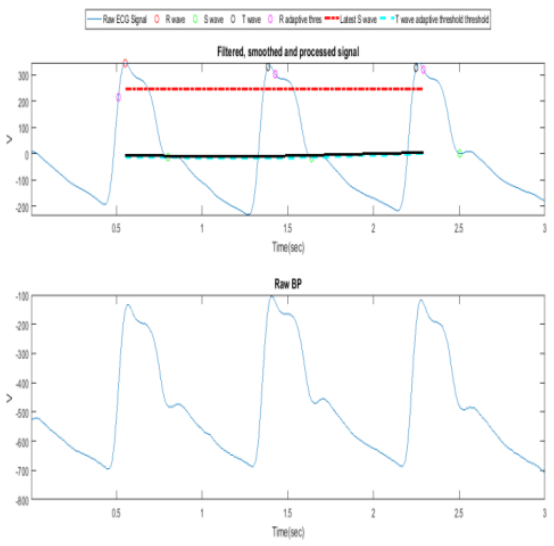

Fig 11: The fused output signal

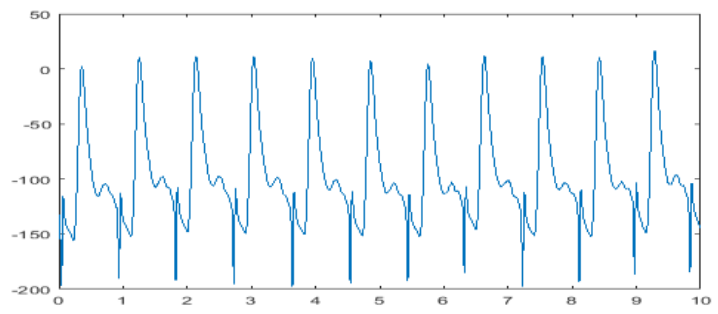

Fig 12: The heart rate obtained from the fused signal
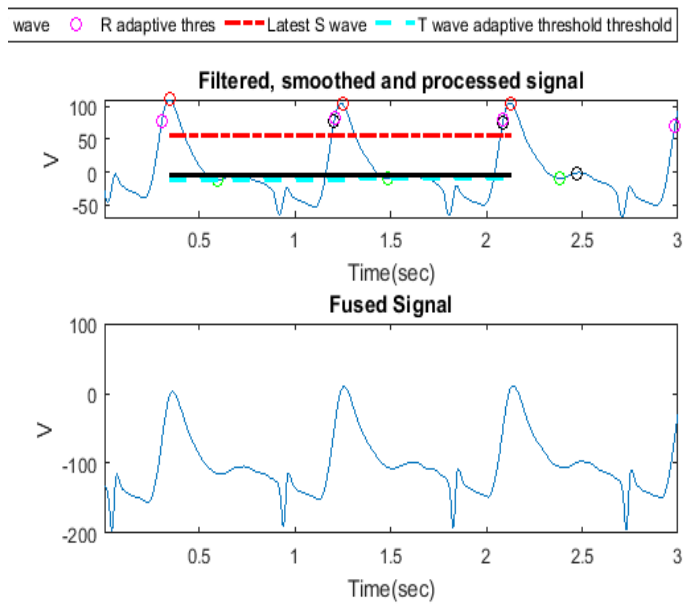

\section{Results and Discussion:-}

Table 1 and Fig 1 shows the correlation of ECG signals with BP, EEG and RR signals separately. It is observed from the graph that there is a significant correlation between ECG and BP only. Though there is a wide variation in EEG, it cannot be chosen because brain waves are not only dependent on heart rate alone but have numerous parameters that affect it. 
Fig 3 represents a pure ECG signal obtained from the sleep database. In order to process the signal as in a real time environment, noise was added represented by fig 4 and $5(\mathrm{SNR}=20 \mathrm{~dB}$ and $40 \mathrm{~dB}$ respectively).Fig 6 represents the heart rate obtained from the ECG signal.

Fig 7 represents a pure BP signal obtained from the sleep database. In order to process the signal as in a real time environment, noise was added represented by fig 8 and $9(\mathrm{SNR}=20 \mathrm{~dB}$ and $40 \mathrm{~dB}$ respectively). Fig 10 represents the heart rate obtained from the BP signal.

Fig11 shows the output waveform of the fusion algorithm. The fusion algorithm $m$ estimates the combination of both the signals. Visually, it shows the merged form of the ECG and BP signals. Fig12 shows the heart rate of the fused signal in the processed form

Table 2: Comparison of HR between original and estimated signal.

\begin{tabular}{|c|c|c|c|c|}
\hline \multirow[t]{2}{*}{ Data/SNR } & \multirow{2}{*}{$\begin{array}{l}\text { Actual HR from } \\
\text { original ECG signal }\end{array}$} & $10 \mathrm{~dB}$ & $20 \mathrm{~dB}$ & 30dB \\
\hline & & \multicolumn{3}{|c|}{ HR obtained by using Kalman fusion algorithm } \\
\hline Slp01 & 66 & 66 & 66 & 66 \\
\hline Slp14 & 72 & 78 & 78 & 78 \\
\hline Slp37 & 60 & 66 & 66 & 66 \\
\hline Slp41 & 66 & 66 & 66 & 66 \\
\hline Slp59 & 78 & 84 & 84 & 84 \\
\hline Sp67x & 72 & 72 & 72 & 72 \\
\hline
\end{tabular}

Table 2 shows the outputs of our project that tabulates the improved heart rates of the various inputs considered. The standard or reference heart rate is the heart rate of the ECG alone. The tabulation shows the heart rates of the fused signals. It can be observed that incase of normal inputs; the estimated heart rate is the same as that for the reference signal. Only in the case of records with certain abnormalities, that there is a difference.

Table 3: Comparison of error in estimating the HR values.

\begin{tabular}{|c|c|c|c|c|}
\hline \multirow[t]{2}{*}{ Data/SNR } & \multirow{2}{*}{$\begin{array}{l}\text { Actual HR from } \\
\text { original ECG signal }\end{array}$} & $10 \mathrm{~dB}$ & $20 \mathrm{~dB}$ & 30dB \\
\hline & & \multicolumn{3}{|c|}{$\begin{array}{c}\% \text { Error }=(\text { Orignal HR-Est HR)/Original HR } * 100 \\
\text { (obtained by using Kalman fusion algorithm) }\end{array}$} \\
\hline Slp01 & 66 & 0 & 0 & 0 \\
\hline Slp14 & 78 & -7.69 & -7.69 & -7.69 \\
\hline Slp37 & 60 & -10.01 & -10.01 & -10.01 \\
\hline Slp41 & 66 & 0 & 0 & 0 \\
\hline Slp59 & 78 & -7.7 & -7.7 & -7.7 \\
\hline Sp67x & 72 & 0 & 0 & 0 \\
\hline
\end{tabular}

Table 3 displays the percentage error for all the signals considered. Percentage error is obtained as the ratio of the original heart rate to the difference between the original and the estimated heart rate. What we can infer from the table is that the maximum error is only $10 \%$, which is quite a reasonable value considering the amount of noise added to the signals for evaluation purposes.

\section{Conclusion:-}

The main purpose of this paper is to obtain the heart rate from the fusion of ECG and BP signals. From the information obtained as the output of the peak detector for the fused signal the estimated heart rate is as shown in the above tables. This implies that fusion of signals gives the heart rate even on a noisy dataset. The added advantage of this approach is that the error value depends on the quality of the signals chosen for the study. In some cases the error is negligible or not present at all, which implies that this approach is headed in the right direction 


\section{Reference:-}

1. ] Susan Redline, Mark H. Sanders,Bonnie K. Lind, Stuart F. Quan,Methods for Obtaining and Analyzing Unattended Polysomnography Data for a Multicenter Study,Researchgate, Accepted for publication August, 1998

2. Zong W, Moody GB, Mark RG. Reduction of false arterial blood pressure alarms using signal quality

3. assessment and relationships between the electrocardiogram and arterial blood pressure. Med Biol EngComput 2004;42:698-706. [PubMed: 15503972]

4. C. O'Brien and C. Heneghan, "A comparison of algorithms for estimation of a respiratory signal from the surface electrocardiogram," Computers in Biology and Medicine, vol. 37, no. 3, pp. 305-314, 2007

5. Clifford GD, Aboukhalil A, Mark RG. Using the blood pressure waveform to reduce critical false ECG alarms. ComputCardiol 2006;33:829-32.

6. Kohler BU, Hennig C, Orglmeister R. The principles of software QRS detection. IEEE Eng Med Biol Mag 2002;21:42-57. [PubMed: 11935987]

7. Hamilton PS, Tompkins WJ. Quantitative investigation of QRS detection rules using the MIT/BIH arrhythmia database. IEEE Trans Biomed Eng 1986;33:1157-65. [PubMed: 3817849]

8. Q. Li, R. G. Mark, and G. D. Clifford, "Artificial arterial blood pressure artifact models and an evaluation of a robust blood pressure and heart rate estimator," BioMedical Engineering Online, vol. 8, article 13, 2009.

9. Q. Li, R. G. Mark, and G. D. Clifford, "Robust heart rate estimation from multiple asynchronous noisy sources using signal quality indices and a Kalman filter," Physiological Measurement, vol. 29, no. 1, pp. 15-32, 2008.

10. Brown, RG. Introduction to Random Signal Analysis and Kalman Filtering. New York: Wiley; 1983

11. Clifford, GD. Linear filtering methods. In: Clifford, GD.; Azuaje, F.; McSharry, PE., editors. Advanced Methods and Tools for ECG Data Analysis. chapter 5. Norwood, MA: Artech House; 2006.

12. Townsend, N. Unpublished Technical Report. Oxford: Department of Engineering Science, University Oxford; 2001. Merging multiple estimates to form a single estimate.

13. Tarassenko L, Mason L, Townsend N. Multi-sensor fusion for robust computation of breathing rate. Electron Lett 2002;38:1314-16.

14. Shamim Nemati, Atul Malhotra,2 and Gari D. Clifford, Data Fusion for Improved Respiration Rate Estimation

15. Received 7 January 2010; Revised 11 March 2010; Accepted 8 May 2010

16. R. Bailon, L. S ' ornmo, and P. Laguna, "A robust method for " ECG-based estimation of the respiratory frequency during stress testing," IEEE Transactions on Biomedical Engineering, vol. 53, no. 7, pp. 1273-1285, 2006.

17. Tarassenko, L.; Townsend, N.; Price, JD. Combining measurements from different sensors. United States Patent Application Number 311250. 2003. http://www.freepatentsonline.com/20030187337.html

18. Ebrahim MH, Feldman JM, Bar-Kana I. A robust sensor fusion method for heart rate estimation. J Clin Monit 1997;13:385-93. [PubMed: 9495291] 